\title{
A Communications Systems Laboratory Course
}

\author{
Zhiyong Liu \\ School of Information and Electrical Engineering, Harbin Institute of Technology (Weihai), Weihai \\ 264209, China \\ liuzhiyong@hitwh.edu.cn
}

Keywords: Communication engineering education; software-defined radio; hands-on experience; communication system.

\begin{abstract}
A new laboratory course in communications system has been developed and implemented for the graduate students. This course first teaches students how to verify the effectiveness and correctness of the designed communication system based on MATLAB by simulation, and then, on the basis of the previous verification, how to implement the designed communication system using software-defined radio (SDR) platform. The main aim of the course is to let students apply their theoretical knowledge from previous courses to design a communication system by themselves, and thus better comprehend the theoretical concepts in communication systems. In contrast to the existing courses, a broader range of subjects to do with communication systems are include in the course, which covers the theoretical aspects, simulation, teamwork ability training and laboratory part. Evaluations based on students' results and opinions demonstrate that the implementation of such a scheme in the course is readily feasible.
\end{abstract}

\section{Introduction}

The great complexity of modern broadband wireless communication technologies makes the process of both teaching and learning these technologies a challenging task. This is mainly due to the fact that immediate relationship between cause and effect cannot be easily seen by the study of pure theory. The efficacy of a course covering many theoretical topics can be increased through hands-on experiments as pointed out in [1]. Therefore, it is strongly desired to develop a flexible laboratory platform to teach a wide variety of wireless communication techniques.

Lately, software-defined radio (SDR) concept has gained significant interest among wireless communications communities [2]. SDR is where all the signal manipulations and processing works in radio communication are done in software instead of hardware. So, the SDR is very suitable as a laboratory platform to verify different communication systems by conveniently modifying the program. In this paper, a communications system laboratory that enables students to understand communication concepts from different perspectives is introduced.

Senior-level undergraduate and graduate communication engineering students are mainly the target of the course. Most of the concepts for communication system are taught in the earlier stages of a communication engineering education. A literature search reveals that some institutions offer laboratory-based courses focusing on other aspects of communication system as well. One of the examples focusing on wireless sensor networks can be found in [3]. An infrared communication system-based laboratory course is introduced in [4]. Some other techniques to teach wireless communications such as projected-based courses and simulations are also available in the literature [5-10]. The communications systems laboratory course introduced in this paper includes a broader range of subjects to do with communication systems. The course covers the theoretical aspects of communications systems, simulation based on MATLAB, teamwork ability training and laboratory part based on SDR.

\section{Description of the Course}

The communications systems laboratory course is a two-credit course designed for graduate students. The theoretical aspects of the course are discussed in ten in-class session, each class lasts 
approximately 2 hours. The basic theory, method and implementation technology of communication system modeling and simulation are discussed among the theoretical issues. Experiments are designed in order to strengthen the student perspective on theoretical materials, and consist of 18 hours. The laboratory provided a computer with software including MATLAB and GNU Radio as a simulation tool, and a universal software radio peripheral (USRP) platform, which consists of two main boards. Fig. 1 shows the typical experiment environment.

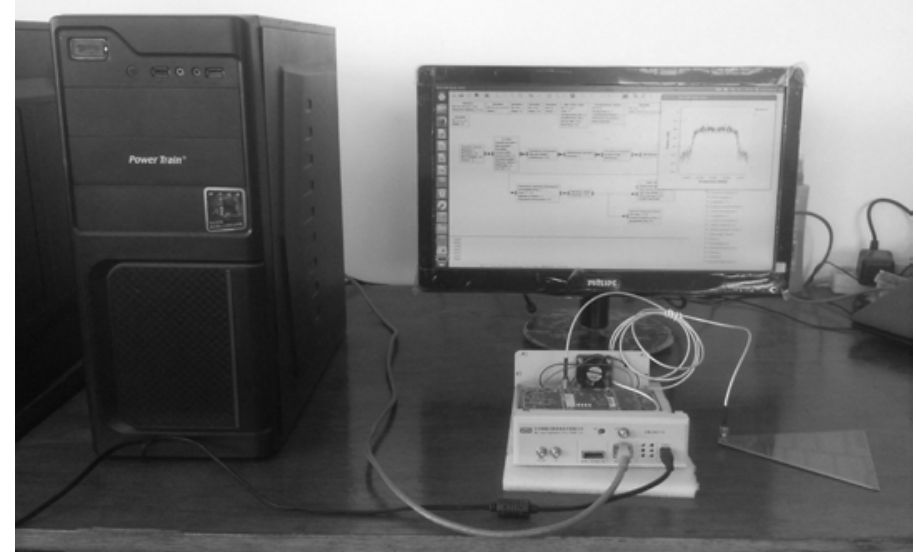

Fig. 1 The typical experiment environment

In the course, teaching and practical scientific research project would be combined in order to train the student's ability of scientific research. The course content is as follows.

\subsection{Theoretical Aspects of Communications Systems Modeling and Simulation}

For the analysis and design of a new communication and signal-processing systems, the simulation-based approach is very important, as a rapid prototyping environment of new system can be created by the approach. However, in order to guarantee the correctness of the simulation, scientific method of simulation must be adopted. Therefore, the teaching content will include modeling and simulation methodology, stochastic process in simulation, Monte Carlo simulation method, random number generation, channel models, communication system modeling etc.

Through learning of the stage, the purpose is to let students master the basic theory, method and implementation technology of communication system modeling and simulation. This is necessary, because even undergoing the studies of undergraduate, but for students, the time of practicing simulation is still limited, they have not systematically studied communication system simulation and modeling knowledge.

\subsection{Teamwork Ability Training}

The complexity of communication and signal processing systems has grown considerably during the past decades. The growth in complexity of communication systems would increase the time and effort required for analysis and design, which make the communication system simulation difficult to be quickly completed by only a person. To speed up the system simulation and develop the student's ability of teamwork, in the course, students will be grouped to cooperatively complete the simulation. In each team, each student will complete one or several module simulation of communication system. The steps used to train ability of teamwork can be outlined as follows:

Table 1 The content of training teamwork ability

\begin{tabular}{cl}
\hline No. & \multicolumn{1}{c}{ Content } \\
\hline 1 & cooperatively analyze the theory of a communication system and algorithm; \\
2 & arrange multiple discussion about the project plan; \\
3 & divide the systems into different modules; \\
4 & arrange multiple internal communication in the team; \\
5 & complete the modeling of each module; \\
6 & $\begin{array}{l}\text { cooperatively complete the whole system simulation based on the model in } \\
\text { previous step; }\end{array}$ \\
7 & $\begin{array}{l}\text { thoroughly analyze their respective modules, make everyone have a deeper } \\
\text { understanding for the system. }\end{array}$ \\
\hline
\end{tabular}




\subsection{Experiment I-DSSS System Simulation based on MATLAB}

Below follows a brief description of the course offered during 2015 dealing with direct sequence spread spectrum (DSSS) communication system. The intent is to illustrate how the case study concept is used to teach system design. The simulation work is done with grouping mode.

Spread spectrum technology has become very important in wireless information networks. In such systems, pseudonoise (PN) code can spread the bandwidth of transmitted signal. Such systems employ CDMA to allow users to share the same wireless radio channel simultaneously. Spread spectrum has many advantages, include its immunity to multipath, ability to suppress interferers, low-power spectral density, efficient use of the RF spectrum, and so on. The experiment describes the complete DSSS transmitter and receiver implementations, as shown in Fig. 2. The aforementioned advantages are also examined. The students would examine in both the time and frequency domains to observe the effect of spreading using the PN code and the dispreading takes place in the receiver when the broad-band received signal is cross correlated with a synchronized replica of the PN code used in the transmitter.

The transmitted signals are usually affected by the interference and broad-band Gaussian noise. By analyzing spectrum of the signal at the output of the receiver's PN code correlator. One can readily observe how the undesired signals are spread in the receiver while the desired signal is despread, demonstrating the power of spread spectrum in suppressing interfering signals and noise. The results of simulation verification can help motivate students and stimulate their interest when studying a particular topic such as wireless communications. The benefit of using a MATLAB environment to teach students the fundamental concepts of spread spectrum system is twofold. First, students can understand more deeply the spread spectrum communication system, which includes the function of each module, the implementation of each module, processing flow and details of signal processing algorithm, and so on. Second, students become familiar with the MATLAB functions and simulation environment upon performing the simulation of DSSS communication system.

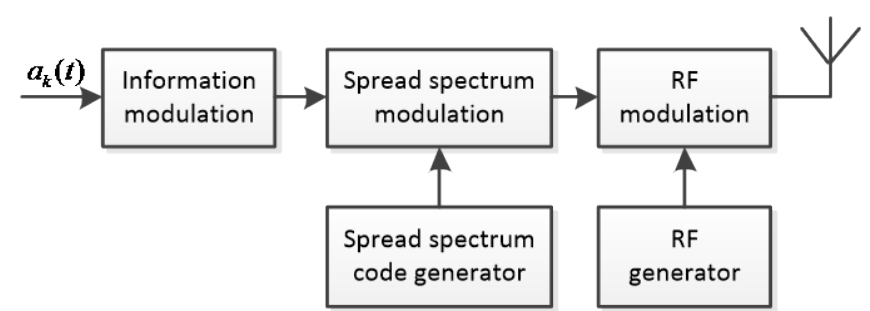

(a) Transmitter

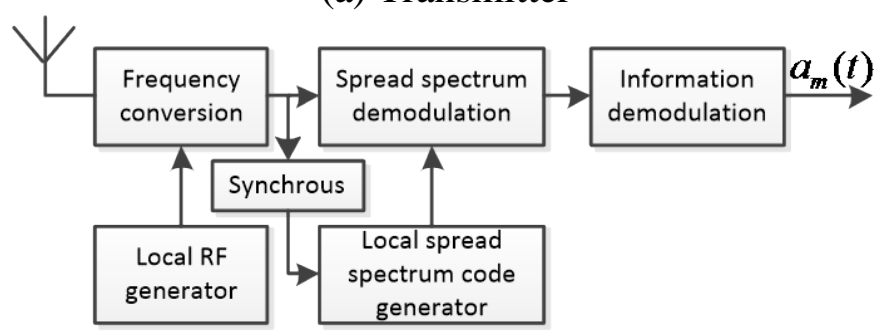

(b) Receiver

Fig. 2 Block diagram of DSSS

\subsection{Experiment II-DSSS System Implementation Utilizing GNU Radio and USRP SDR}

In conventional radio, all the processes which include demodulation, channel coding, source coding and etc. But for SDR, it is defined as a software based communication platform which characteristics can be reconfigured and modified to perform different functions at different times. Moreover, GNU Radio is an open source software toolkit which consists of signal processing blocks library and the glue to tie there blocks together for building and deploying SDR. Therefore, GNU Radio and USRP SDR are very suitable as a low-cost teaching platform to allow students to verify different communication systems and algorithms. 
The experiment II is carried out on the basis of experiment I. For the implementation of DSSS in GNU Radio, the mentioned blocks in Fig. 2 are required. These blocks along with other inbuilt blocks are connected in a particular manner according to the applications. Blocks in GNU Radio are written in $\mathrm{C}++$ while Python is used as a scripting language to tie the blocks together to form the flow graph. Simplified Wrapper and Interface Generator (SWIG) is used as the interface compiler which allows the integration between $\mathrm{C}++$ and Python language. The experiment contents are arranged as following:

Table 2 The content of experiment II

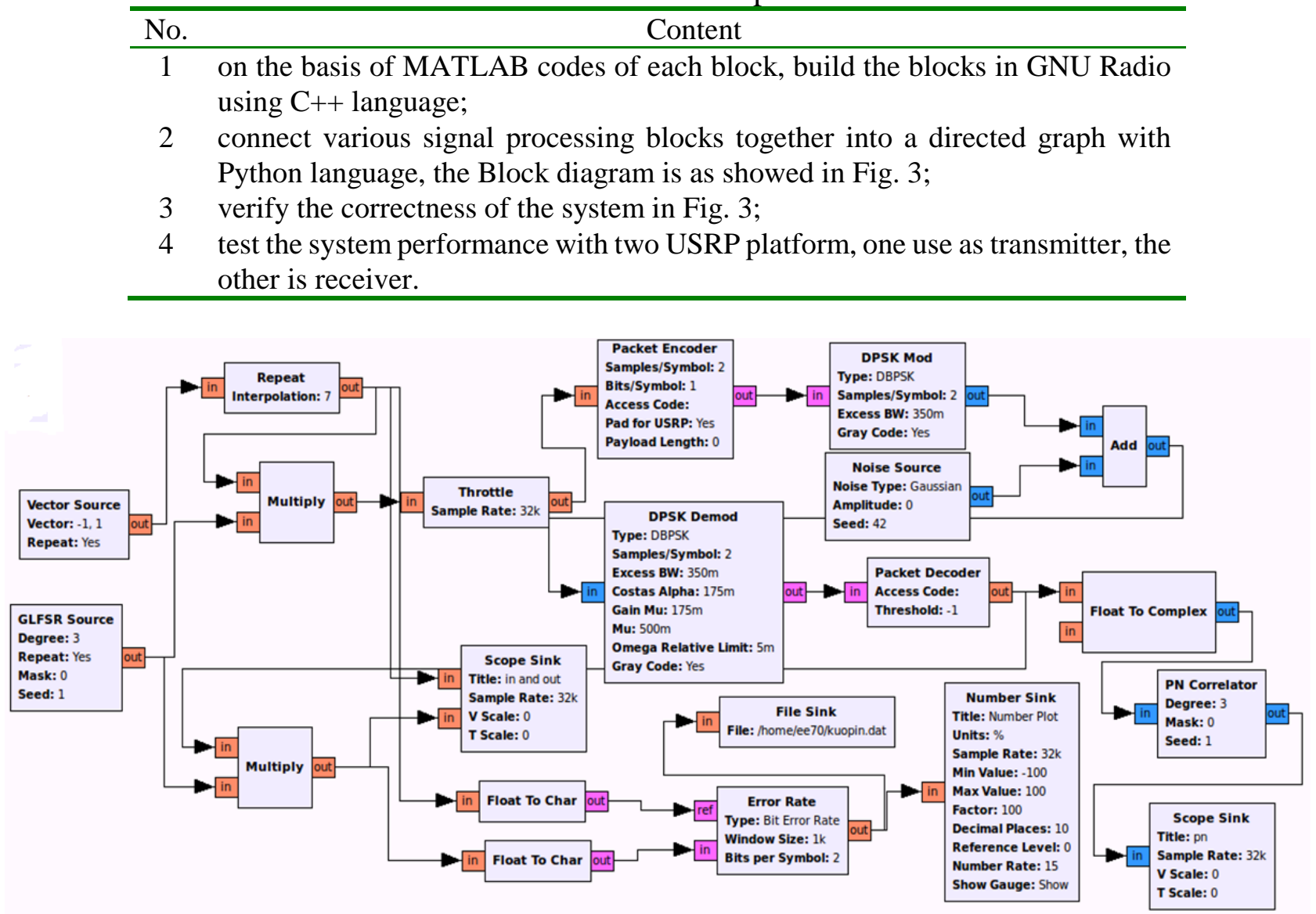

Fig. 3 Block diagram of DSSS in GNU Radio

In addition, we also can use a spectrum analyzer to observing the actual signal, verify the advantages of DSSS. The experiment II is usually met with surprise and excitement on the part of the students, because they build own system, and observe the correct communication through wireless channel between two USRP. The results will stimulate their interest when studying a particular topic such as wireless communications, and make them change learning attitudes from passive learning to autonomous learning.

\section{Assessment}

All students are evaluated based on three qualitative criteria: the realization degree of the whole system simulation, the realization degree of module completed by respective student, and responses to teacher's questions, each of which is rated at $40 \%, 30 \%$, and $30 \%$ of the total grade, respectively. Grades are finalized in an evaluation meeting during the examination period. The final conclusion is based on the three presentations, and a final written report.

The realization degree of the whole system simulation is to judge the entire group's achievement in building the whole system as initially planned. The realization degree of module completed by respective student is based on their success in accomplishing their respective task. Additionally, the ability of teamwork is also assessed on teacher's observation, the final report and presentations. The 
responses to teacher's questions are based on the situations that each student answers the question, which reflects the extent to which they have a grasp of their responsible work.

\section{Conclusion}

This paper presented a successful implementation of a communications systems laboratory course in communication engineering. A combination of the theoretical aspects, ability of simulation, teamwork training, and hands-on ability within this course can cultivate student's research ability, help students to develop good research habits.

The course was found to be very motivating and interesting by the majority of the students. Evaluation of the course is very encouraging as well. The content of experiments and the methodology to keep students motivated can be very inspiring and useful for other institutions.

\section{Acknowledgment}

The work was supported by the Graduate Education and Teaching Reform Research Project in Harbin Institute of Technology (JGYJ-201625) and Postgraduate education innovation program of Shandong Province (SDYC15018).

\section{References}

[1]. M. F. Young, “Instructional design for situated learning,” Educ. Technol. Res. Develop., vol. 41, no. 1, pp. 43-58, Mar. 1993.

[2]. Marwanto, A., Sarijari, M. A., Fisal, N., Yusof, S. K. S., \& Rashid, R. A., "Experimental study of OFDM implementation utilizing GNU radio and USRP-SDR,” In Communications (MICC), 2009 IEEE 9th Malaysia International Conference on, pp. 132-135, Dec. 2009.

[3]. Frolik and M. Fortney, "A low-cost wireless platform for first-year interdisciplinary projects," IEEE Trans. Educ., vol. 49, no. 1, pp. 105-112, Feb. 2006.

[4]. W. Padgett, B. Black, and B. Ferguson, "Low-frequency wireless communications system-infrared laboratory experiments,” IEEE Trans. Educ., vol. 49, no. 1, pp. 49-57, Feb. 2006.

[5]. N. Sarkar and T. Craig, "Teaching wireless communication and networking fundamentals using Wi-Fi projects,” IEEE Trans. Educ., vol. 49, no. 1, pp. 98-104, Feb. 2006.

[6]. P. Shankar and B. Eisenstein, "Project-based instruction in wireless communications at the junior level,” IEEE Trans. Educ., vol. 43, no. 3, pp. 245-249, Aug. 2000.

[7]. G. Prabhu and P. Shankar, "Simulation of flat fading using Matlab for classroom instruction," IEEE Trans. Educ., vol. 45, no. 1, pp. 19-25, Feb. 2002.

[8]. Aliakbarian H., Soh P. J., Farsi S., et al, "Implementation of a project-based telecommunications engineering design course,” IEEE Transactions on Education, vol. 57, no. 1, pp. 25-33, Feb. 2014.

[9]. Warin B., Talbi O., Kolski C., et al, "Multi-Role Project (MRP): A New Project-Based Learning Method for STEM,” IEEE Transactions on Education, vol. 59, no. 2, pp. 137-146, May. 2015.

[10]. Rashid M., “System Level Approach for Computer Engineering Education,” INTERNATIONAL JOURNAL OF ENGINEERING EDUCATION, vol. 31, no. 1, pp. 141-153, 2015. 